\title{
Rheumatoid Arthritis: Treatment and Pharmacological Therapies
}

\author{
Midhun Kumar G.H. ${ }^{1}$, Shanmuga Priyan ${ }^{2}$, Arunachalam P. ${ }^{3}$, Elavarasan K. ${ }^{4}$ \\ ${ }^{1}$ Associate Professor, Department of Community Medicine, Meenakshi Medical College Hospital and Research \\ Institute, Enathur, Kanchipuram, Tamilnadu, India, ${ }^{2}$ Associate Professor, Department of Pharmacology, Meenakshi \\ Medical College Hospital and Research Institute, Enathur, Kanchipuram, Tamilnadu, India, ${ }^{3}$ Professor \& HOD, \\ Department of Cardiology, Meenakshi Medical College Hospital and Research Institute, Enathur, Kanchipuram, \\ Tamilnadu, India, ${ }^{4}$ Senior Resident, Department of General Medicine, Meenakshi Medical College Hospital and \\ Research Institute, Enathur, Kanchipuram, Tamilnadu, India
}

\begin{abstract}
Arthritis is a persistent autoimmune ailment occurs more in females than in male above the age of 40 . It initially affects the lining of synovial joint and the secretion of low synovial fluid also can lead to rheumatoid arthritis. It primarily affects the patient's health swelling in joint, redness and level decreases eventually. Diagnosis at an early stage of rheumatoid arthritis is the key to prevent from severe injuries in joint and other bone related diseases. There is also several plant based anti-inflammatory compound which involves in decreasing the pathogenesis of the ailment. There are several diseases related to rheumatoid arthritis, it includes pulmonary granuloma, vesicle damages and other symptoms are expected to occur early if unnoticed. This article discusses the risk factors, damages to joint and pharmacological therapies available for the diseases.
\end{abstract}

Keywords: Arthritis, Pharmacology, synovial joint, pathogenesis, anti-inflammatory.

\section{Introduction}

Rheumatoid arthritis or synovial hyperplasia is a provocative disease, occurs to damage the properties of joints. $T$ cells, endothelial cells and antigen presenting cells that are majorly involved in the rheumatoid arthritis $^{[1]}$. An addition to the inflammatory cells participation fibroblasts produced in the synovial membrane play an important part in the complications of RA. These fibroblasts exhibit the constant cellular activation, which is processed routinely in the nonappearance of inflammatory stimuli. In osteoarthritis

\section{Corresponding Author:}

Midhun Kumar G.H.

Associate Professor, Department of Community

Medicine, Meenakshi Medical College Hospital and

Research Institute, Enathur, Kanchipuram, Tamilnadu, India

e-mail: midhunkumargh@mmchri.ac.in patients, the synovial fibroblast shows the gene expression of proto oncogenes and its transcriptional factors which helps in the degradation of and it ends up with apoptosis. Here the complications develop as these fibroblasts stick to the cartilage to increasingly destroy the articular structures. The mechanism of RA is that it leads to the constant activation synovial fibroblast development. Dietary intake in a healthy manner contributes to the reduction of RA also having chance to reduce the occurrence of this disease at the young age. Hormonal levels are noticed in the pathogenesis of RA. By observing the above said complications, it is important to find the effective treatment strategies and therapies for rheumatoid arthritis would be essential ${ }^{[2]}$.

\section{Therapies for Rheumatoid Arthritis:}

Methotrexate: The diagnosis of the disease, Methotrexate is prescribed by the doctors as a first line of defense and it is usually prescribed with 15$20 \mathrm{mg}$ of the week and gradually increased up to $25 \mathrm{mg}$ per week. It is usually as a combination medicine with 
glucocorticoids to compensate the mechanism. The production of this medicine is limited and based on the availability it is combined with other medicines. The combinations mainly results in adverse effects in patients when increasing the rate of administration. Increased level of dosage in patients sometimes leads to failure of methotrexate reaction because of the risk associated with this disease ${ }^{[3]}$. The long term study on this compound for RA has showed highest recovery rate. It is reported in research articles that among the rheumatologists in the U.S methotrexate has a good recovery rate for RA also for other diseases ${ }^{[4]}$.

\section{RITUXIMAB}

Rituximab a monoclonal antibody anti-CD20 recognized for the treatment of a rheumatoid arthritis. Bone marrow is located where B-cells are produced and it is an impactful therapy against the disease after the effective treatment with rituximab. The patients who took this compound have been observed in the clinical response later they were implemented with the B-cell treatment. Association of these two therapies helps the patients with longtime maintenance of the patients with RA. The effect of this treatment results in the production of protein and rheumatoid factor helps the patients in recovery of the disease. Patients treated with this therapy before the rituximab shows slow clinical response for RA. Anyhow, half of the patients regain their effect for the treatment with B-cell depletion therapy after the treatment with rituximab. Patients are observed for the risk development. The risks occur due to the dosage level of rituximab and people are prone to infection. The most common adverse effects are the level of $\operatorname{IgG}$ concentration associated with the rheumatoid arthritis disease $^{[5]}$.

Anti-TNF Therapies: This TNF therapy was identified in the year 1980's for the pathogenesis of rheumatoid arthritis in animals and also in human. The evidence from this impact is studies in mice with TNF expressing factors. It revealed that the mice induced with rheumatoid arthritis could be prevented by stopping the production of TNF-alpha factor. There are three drugs mainly inhibiting the TNF are infliximab was testes in RA patients with clinical approval and the next is an Adalimumab antibody against the TNF factor and finally etanercept which was biologically approved to treat RA. Patients were improved after the treatment with these drugs. The TNF factor block was the major challenging in the improvement of treatment with $\mathrm{RA}^{[6]}$.
Synovial Fibroblasts: The recovered rheumatoid arthritis patients were incorporated with synovial fibroblasts, which are present in rheumatoid synovium, which are considered as the destructor of causes for rheumatoid arthritis. After the incorporation it releases the increased amount of enzymes that prevent the cells from direct contact with RA cells. The endothelial cells that tend to act as the destroyer of articular cartilage. These are the ways how it recruits the cells to fight the RA with the help of macrophages, and T-cells and the rheumatoid synovium that attracts large amount of inflammatory cells that develops the activation of osteoblasts ${ }^{[7]}$.

Cytokines: The activity level of cytokines is based on the secretion and the target of cell for the destruction. It clearly indicates that the response to inflammatory stimuli is just developed by the production of competing cells that are antagonists. Research studies have reported that the existence of polymorphisms of the TNF, and IL-Ira genes. Particularly the TNF-gene is situated on the chromosome 6 short arm in the HLA region. The abnormal high molecular weight TNF mRNA has been observed in the macrophages of murine and in monocytes of human that are infected with influenza A virus ${ }^{[8]}$.

Eicosanoids and Lymphokines: These two immune agents are known to present in the inflammation process. In this process the lymphocytes and macrophages are activated to produce this agent eicosanoids which have higher efficiency to work against inflammatory cells. These two compounds can modulate the immune responsiveness. Proteases and collagenases which are known to degrade the secretion of cells and bone development which are related to many vascular diseases in the bone related diseases. TGF (Tumor growth factor-beta) produced by synovial tissues and lymphocytes can stop the process of inflammation. Fatty acids are the suppressors of T-cell proliferation. The major interleukins such as 1, 2 and the production of TNF are shown to have benefit in rheumatoid arthritis patients ${ }^{[9]}$. Rheumatoid arthritis with cognitive issue are related to skills in stress management and other symptoms. It is predicted that the treatment will show a reduction in symptoms and improve both immunological competence and the functioning of bones. The enhanced self-efficient, reduces pain and inflammatory cell proliferation in patients with RA ${ }^{[10]}$.

IL-6: Interleukin-6 is one of the most potential therapeutic target pleiotropic proinflammatory cytokines. 
It is produced by various cells, including lymphocytes, monocytes, and fibroblasts. It is involved in the multiple immunological processes of the immune cell activation. These cells are particularly in relevance with Rheumatoid arthritis, interleukin-6 induces osteo cell differentiation and it contributes to joint osteoporosis ${ }^{[11]}$.

Celastrol: The other bioactive component of TwHF, celastrol belongs to celastraceae family it has many antiarthritic activity. The research studies have revealed that it can suppress that rheumatoid arthritis severity in the AA Lewis rat model. Moreover, it can suppress the proinflammatory cytokines production and balance Thymus cell to regulate the morphology in the targeted organ. Additionally, celastrol compound protects the erosion of bone by targeting certain pathways and the deviating osteoprotegerin level stops the osteoclastic activity which in turn reduces the damage in the bones ${ }^{[12]}$.

Curcuma longa LINN: Curcuma is the anti-potent agent which grows in tropical regions, mainly in India and China. The turmeric heated with oil and applied all over the knees. The chemical compounds present in this plant are Sodium-Curcuminate, Curcumin and Methylcurcumin. In our tradition, it is used for healing antiseptic and possesses anti-helminthic activity. It is studied that herbal formulation produced from the turmeric plant works well with the arthritic patients. It is researched to possess anti-inflammatory activity, ant-fungal, anti-protozoal activity and hepatoprotective agents. The oil produced from the plant has high antiinflammatory activity and anti-arthritic activity ${ }^{[13]}$.

Inula Helenium: It is a perennial plant also known as horse heals. Importantly dihydroflavonols elicited from the aerial part of the plant showed antiinflammatory activity. This compound significantly suppresses the activity in rat peritoneal leukocytes. These flavonoids are the components of viscosa plants reduce the leukotriene production. Elastin is the outer cell protein, has the automatic effect on dissimilar tissues. Hence it is referred as the contributory agent in the reduction of $\mathrm{RA}^{[14]}$.

Pluchea lanceolata: This plant is one of the important medicinal plant having these anti-inflammatory and analgesic activities for rheumatoid arthritis, sciatica, edema and psoriasis. This involves the synthesis of different secondary metabolites viz., flavonoids and daidzein and the major compound pluchine from this plant contributes in the anti-inflammatory activity. The alcohol extract from pluchea plant showed maximum reduction in formalin induced arthritis in rats. The powder form of the plant is applied for arthritis ${ }^{[15]}$.

Madimadi: It is a Korean based medicinal plant, fermented alcohol prepared from the extracts of Madi madi. It is found to be the curative agent for long time in the tradition of Korean medicines. It has an inhibition effect on the pro-inflammatory cytokine and dose dependent inhibition against of patients with RA with these interleukin-beta and interleukin-8. It also down regulates the alpha-tumor necrosis factor. It is a recurrent plant with creeper like structure found growing in Taiwan. The main active compound present is called triptolide has anti-inflammatory activity ${ }^{[16]}$.

\section{Conclusion}

Rheumatoid arthritis occurs mostly in aged patients with many health complications such as swelling of knee, redness and motion reduction in the bones. The synovial fluid synthesis is the major cause of arthritis. It can be cured with many therapeutic agents when treated early with worthy medications. Some of the important plants also contribute to the anti-inflammatory activity, neurological disorders and patients suffering from sciatica. Monoclonal antibodies based therapies are most welcome in people from developing countries with highest medicinal facility. Still medicinal plant based drugs are mostly preferred for the cure because of its $\log$ term effects. This paper presented some of the important therapeutic agents both synthetic and traditional medicines for the patients with rheumatoid arthritis complications.

\section{Ethical Clearance: Nil}

Source of Funding: Meenakshi Academy of Higher Education and Research, Chennai, India

\section{Conflict of Interest: Nil}

\section{References}

1. Guo Q, Wang Y, Xu D, Nossent J, Pavlos NJ, Xu J. Rheumatoid arthritis: pathological mechanisms and modern pharmacologic therapies. Bone research. 2018 Apr 27;6(1):1-4.

2. Müller-Ladner U, Pap T. Pathogenesis of RA: more than just immune cells. Zeitschrift fur Rheumatologie. 2005 Sep;64(6):396.

3. Köhler BM, Günther J, Kaudewitz D, Lorenz HM. 
Current therapeutic options in the treatment of rheumatoid arthritis. Journal of clinical medicine. 2019 Jul;8(7):938.

4. Weinblatt ME. Methotrexate in rheumatoid arthritis: a quarter century of development. Transactions of the American Clinical and Climatological Association. 2013;124:16.

5. Garcia-Montoya L, Villota-Eraso C, Yusof MY, Vital EM, Emery P. Lessons for rituximab therapy in patients with rheumatoid arthritis. The Lancet Rheumatology. 2020 May 12.

6. Josef S SmolenGünter Steiner (2003), Therapeutic strategies for Rheumatoid arthritis Article:Nature Reviews Drug Discovery.

7. Nogueira E, Gomes A, Preto A, Cavaco-Paulo A. Update on therapeutic approaches for rheumatoid arthritis. Current Medicinal Chemistry. 2016 Jun 1;23(21):2190-203.

8. Campion GV. The prospect for cytokine based therapeutic strategies in rheumatoid arthritis. Annals of the rheumatic diseases. 1994 Aug;53(8):485.

9. Das UN. Interaction (s) between essential fatty acids, eicosanoids, cytokines, growth factors and free radicals: relevance to new therapeutic strategies in rheumatoid arthritis and other collagen vascular diseases. Prostaglandins, Leukotrienes and Essential Fatty Acids. 1991 Dec 1; 44(4):20110.

10. Rheumatoid arthritis and other collagen vascular diseases: Prostaglandins, Leukotrienes and Essential Fatty Acids.

11. O’Leary A, Shoor S, Lorig K, Holman HR. A cognitive-behavioral treatment for rheumatoid arthritis. Health Psychology. 1988;7(6):527.

12. Dudics S, Langan D, Meka RR, Venkatesha SH, Berman BM, Che CT, Moudgil KD. Natural products for the treatment of autoimmune arthritis: their mechanisms of action, targeted delivery, and interplay with the host microbiome. International journal of molecular sciences. 2018 Sep;19(9):2508.

13. Choudhary M, Kumar V, Malhotra H, Singh S. Medicinal plants with potential anti-arthritic activity. Journal of intercultural ethnopharmacology. 2015 Apr;4(2):147.

14. Farzaei MH, Farzaei F, Abdollahi M, Abbasabadi Z, Abdolghaffari AH, Mehraban B. A mechanistic review on medicinal plants used for rheumatoid arthritis in traditional Persian medicine. Journal of Pharmacy and Pharmacology. 2016 Oct;68(10):1233-48.

15. Bagde S, Biswas D. A review on some selected traditional Indian medicinal plants of immunomodulatory potential and their therapeutic use in rheumatoid arthritis. Int J Pharm Sci Res. 2019;10(5):2087-100.

16. Rathore B, Mahdi AA, Paul BN, Saxena PN, Das SK. Indian herbal medicines: Possible potent therapeutic agents for rheumatoid arthritis. Journal of Clinical biochemistry and Nutrition. 2007 Jul; 41(1):12.

17. Marrelli M, Amodeo V, Perri MR, Conforti F, Statti G. Essential Oils and Bioactive Components against Arthritis: A Novel Perspective on Their Therapeutic Potential. Plants. 2020 Oct;9(10):1252. 